\title{
The Influence of Laser for Laser Ultrasonic Pipeline Detection Analysis
}

\author{
Min $\mathrm{Ji}^{1, a}$ and Jingfeng $\mathrm{He}^{1, \mathrm{~b}}$ \\ ${ }^{1}$ Xijing University, Shaanxi Xi'an, 710123, China \\ a995010771@qq.com, b573825625@qq.com
}

Keywords: Laser ultrasonic; Waveform; Pipe laser; Parameters

\begin{abstract}
This paper mainly introduces the basis of principle of laser ultrasonic detection. Then the different laser parameters adjustment in pipeline detection, observe different waveform display. Finally the corresponding conclusions. In order to provide a basis for later testing.
\end{abstract}

\section{Introduction}

Laser Ultrasonic visualization detector referred as "LUVI, is instrument [1]by the Xi'an jin-bo testing instrument co. LTD and Japan tsukuba that joint research and development of testing instrument. Laser ultrasonic visualization testing systems [2] is simple structure, small volume, high degree of visualization, its composition of four modules.

The main use of pulsed laser irradiation is local suddenly heated surface and expansion, resulting in a thermal elastic wave, it is a kind of ultrasonic wave, like water waves generated by ultrasonic in work piece internal waves forward propulsion. The ultrasonic transmission, through the measured surface by recording and processing software, the propagation record can be in the process of testing the watch at the same time, also we can watch after testing.

When encountered defects, defect reflection will make forward wave abnormalities, back to defect point as the center, to produce ultrasonic. The detection of ultrasonic propagation in the process of shape can be directly see, through the defect echo and the work piece tested different ultrasonic wave, can locate damage, defect of work piece [4].At the same time, we could detect wave transmission by watching animation, combined with the maximum amplitude image to locate the defects exist, its detection principle [3] is shown in Fig. 1.

As the detection of the test process and automatic recording, and it can been shown over and over again. Defects of direct display, and do not need to carry on the processing analysis. The results without moving the probe, so test conveniently, fast, high precision [5].In addition, the non-contact laser ultrasonic detection technology makes it can be applied to a lot of special environment, such as toxic, strong radiation fitting test in service under the bad environment, so you can ensure normal operation of the pipeline, can also be on-line detection, ensure the smooth progress of the safety test.

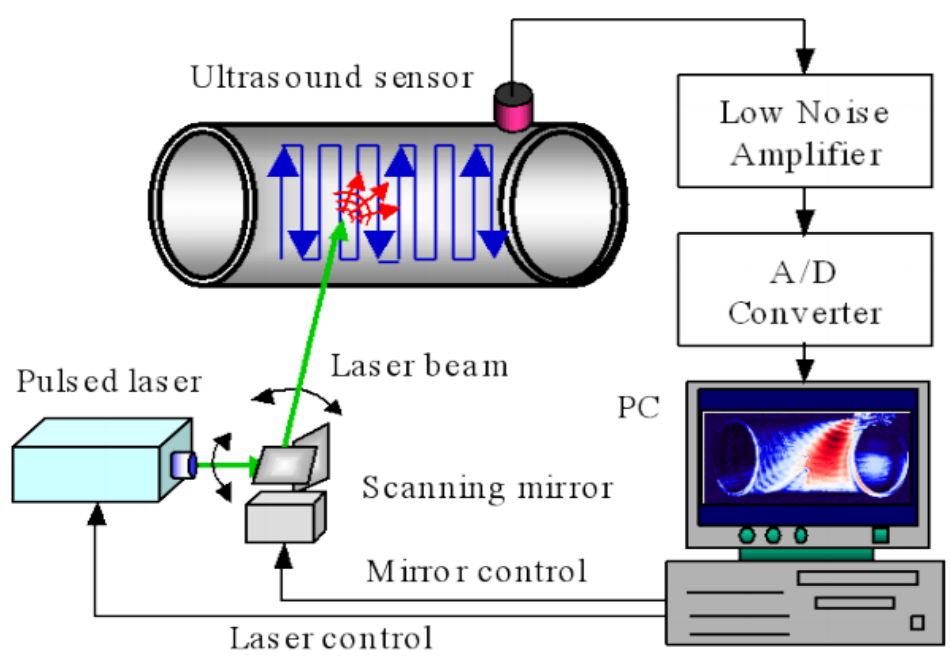

Figure 1. Laser ultrasonic system for visualizing ultrasonic propagations 


\section{The Test Object}

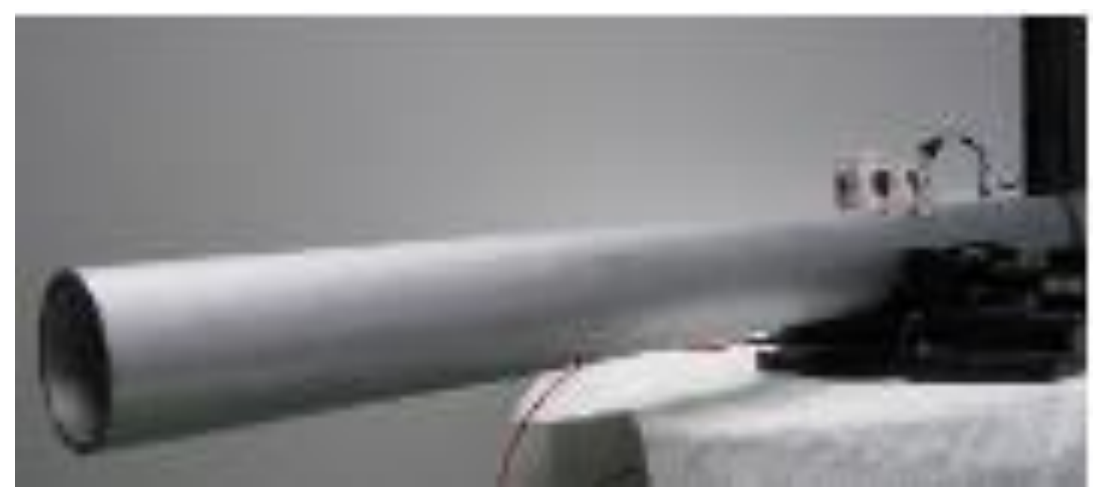

Figure 2. Oil pipeline specimen

Parameter Settings. Probe: $1 \mathrm{MHZ} 90^{\circ}$; Distance: $\mathrm{L}=500 \mathrm{~mm}$; BPF $=1 \mathrm{MHZ}$.

\section{The Incident Laser Angle}

The Experimental Contents. Scanning interval is $0.8 \mathrm{~mm}$, scanning point is $240 \mathrm{x}$ 106.In different incident Angle of laser [6] is shown in figure 2 oil pipeline testing, it can be seen by comparing the analysis Angle influence on test results. Here, with laser beam perpendicular to the pipe axis positioning 00 incident, with 450 respectively after the incident, the incident 600, 700 incident.

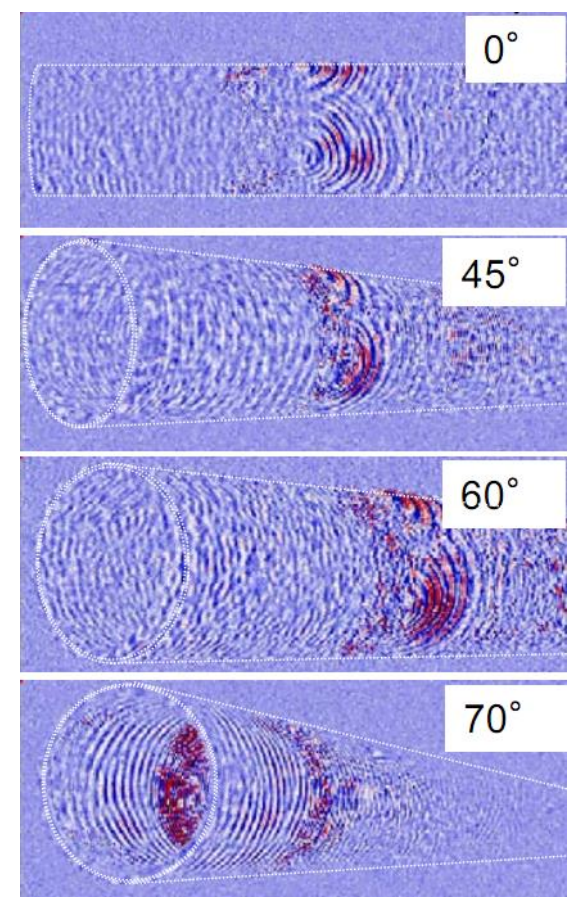

Figure 3. Different angles of incidence laser waveform

The Experimental Results. Compare the above several ways to visualize, as shown in Fig. 3.By figure can be concluded that to 600 is still the echo of the damage can be observed, but by the 700 can see light point to long elliptic, laser irradiation area increases, so the same laser energy per unit area of the work piece under large signal is abate, the resulting ultrasonic signal also weakens, leading to the damage degree of visibility of echo, and even can't see. Continue to increase angle, the detection effect possible distortion, or detect damage signal, seriously affect the accuracy. 


\section{The Laser Scanning Speed (Scanning Frequency)}

The Experimental Contents. Scanning interval is $0.8 \mathrm{~mm}$, scanning point is $240 \times 106$. The scanning speed of laser with different oil pipeline shown in the Fig. 2, through the comparative analysis shows that scanning speed on the result of testing.

The Experimental Results. Compare the above several ways to visualize, as shown in Fig. 4.By the figure can be concluded that the different scanning speed will influence the incentive scan cycle [7].The faster scanning speed, the more incentive cycle short. Especially in testing, when the great work, also cannot blindly in order to shorten the cycle and improve the speed, can be seen from the figure 4 wave forms, with the speeding up of the scanning speed, scanning results clarity also drops. So, choosing the appropriate scanning speed, for general work piece detection is very important, usually we choose between $100-500 \mathrm{~Hz}$ is advisable. And some of the larger work piece can properly through the scanning speed, but the biggest scanning rate of $2000 \mathrm{~Hz}$.
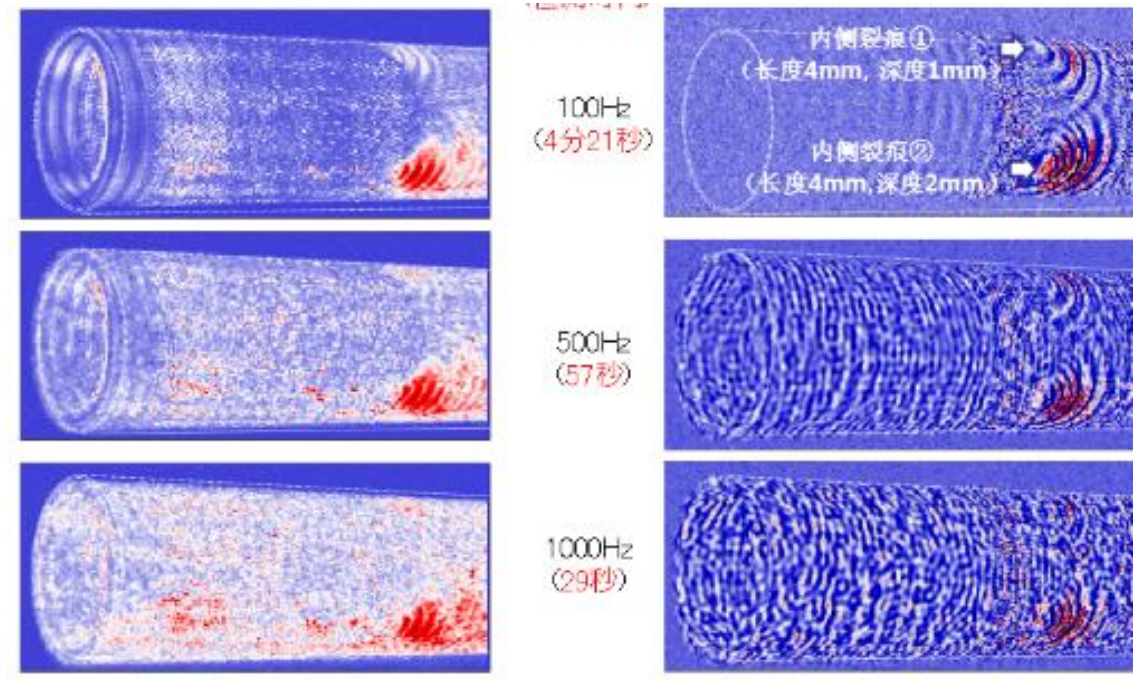

Figure 4. Different scanning speed waveform

\section{The Laser Scanning Interval (Scanning Points)}

The Experimental Contents. Sweep frequency is 200MHZ, laser with different scanning interval of oil pipeline testing is shown in Fig. 2, through the comparative analysis can be seen that the scanning interval on the result of testing [8].

The Experimental Results.
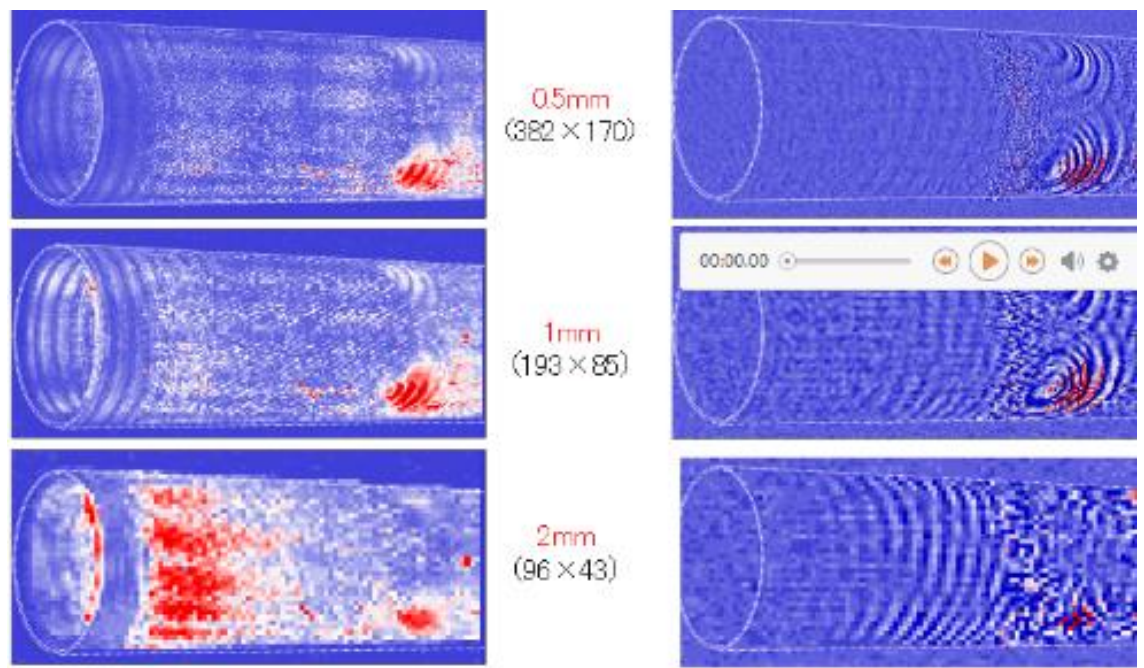

Figure 5. Different scanning interval waveform 
Compare the above several ways to visualize, as shown in Fig. 5. By the figure can be concluded that the different scanning interval also affect the test results. Scan interval is smaller, waveform figure shows the clearer, but also can't infinite narrow scanning interval, our system within a resolution of 60000 points, if more than this range, the signal will be distorted, it will affect the accuracy of detection.

\section{Summary}

Effect analysis of the effect of laser incentive for testing, so that we can to different detection objects, adjust their corresponding parameters, the Angle of incidence, obtain ideal detection results.

\section{References}

[1] B. Wang, J. Takatsubo, et al., "An Improved Ray Tracing Algorithm for Ultrasonic CT in Nondestructive Inspections", Proceedings of the 8th international Conference on Signal Processing, Vol.1, pp5-8. Nov. 2006 in Guilin, CHINA.

[2] H. Yamawaki and T. Saito, "Numerical calculation of ultrasonic propagation with anisotropy", NDT \& E Int., Vol.33, Issue7, 2000, pp489-497.

[3] B. Wang.Laser ultrasonic visualization detector using manual [M]. Tsukuba: Japan tsukuba science and technology co., LTD, 2010.

[4] J. Takatsubo, B. Wang, H. Tsuda and N. Toyama, "Generation laser scanning method for the visualization of ultrasounds propagating on a 3-D object with an arbitrary shape (in Japanese)", Trans. of the Jpn. Soc. of Mech. Engineers, Series A, Vol.72, No.718, 2006, pp945-950.

[5] BAHADUR S, Badruddin R. Erosion particle characterization and the effect of particle size and shape on erosion [J]. Wear, 1990 (138):1892208.

[6] Bo Wang, Junji Takatsubo, et al. The Development of Laser Ultrasonic Visualization Equipment and it Application in Nondestructive Inspection. [C] Proceedings of the of 17th World Conference \& Exhibition on Nondestructive Testing. CHINA, Shanghai, 2008, Nov. 27-31.

[7] Junji Takatsubo, Bo Wang, et al. Generation Laser Scanning Method for the Visualization of Ultrasounds Propagating on a 3-D Object with an Arbitrary Shape [J].Journal of Solid Mechanics and Materials Engineering, 2007, 12: 1405-1411.

[8] Nan yao. The application and development of laser ultrasonic technology [J]. Journal of electrical engineering, 2010, 03. 